\title{
A Polynomial Subalgebra of the Cohomology of the Steenrod Algebra
}

By

Andreas Zachariou

\section{Introduction}

Let $A$ be the mod 2 Steenrod algebra and $H^{* *}(A)=\operatorname{Ext}_{A}^{* *}\left(Z_{2}, Z_{2}\right)$ its cohomology. The ultimate aim in studying $H^{* *}(A)$ is the long-standing problem of computing the homotopy groups of spheres via the Adams spectral sequence $[1] . \quad H^{s, t}(A)$ has been computed up to certain values of $t-s$ by Adams [4], Ivanovskiǐ [6] (see also: International Congress of Mathematicians, Moscow 1966), Liulevicius [8], May [12, 13], Tangora [21]. It is of interest to know any "systematic" phenomena in $H^{* *}(A)$. In this direction a polynomial "wedge" subalgebra of $H^{* *}(A)$ has been obtained by Mahowald and Tangora [9]. Also: Margolis, Priddy and Tangora proved in [10] that the Mahowald-Tangora "wedge" subalgebra is repeated every 45 stems, under the action of a specific "periodicity" operator. The present writer has described shortly in [24] a polynomial subalgebra of $H^{* *}(A)$ generated by $d_{0}, e_{0}, g$. This subalgebra will be described here in more detail. The basic technique is to study $H^{* *}(A)$ by studying $H^{* *}(B)$ for a suitable subalgebra $B$ of $A$. This technique is due to Adams [3]. It has also been used by Margolis, Priddy and Tangora [10]. Moreover G.W. Whitehead [22] shows that, using the Adams technique, one can obtain many polynomial subalgebras of $H^{* *}(A)$.

The present paper is organized as follows: In Section 2 we state the main theorem and sketch its proof. The detailed proof involves the Adams technique and the construction of the generators $d_{0}, e_{0}, g$. These constructions use known relations between the classes $h_{i}$ (see Adams [2], Novikov [19]); they also use Steenrod $\cup_{i}$-products in $F\left(A^{*}\right)$. In Section 3 we describe briefly these cup-i-products and in Section 4 we give the

Communicated by N. Shimada, October 18, 1972. 
actual construction of the generators $d_{0}, e_{0}, g$. These and other generators can also be constructed with the aid of explicit resolutions (see [11], [12], [22]).

The contents of this paper constitute a major part of my doctoral thesis [25] at the University of Manchester, written under the supervision of Professor Adams. I wish to express my sincere thanks to Professor Adams for his suggestions and helpful ideas and for his constant interest and encouragement.

\section{The Main Theorem}

Let $A$ be the mod 2 Steenrod algebra and $H^{* *}(A)$ its cohomology. The following theorem is our main result.

Theorem. $H^{* *}(A)$ contains a polynomial subalgebra generated by the elements $d_{0}, e_{0}, g$. of dimensions $(4,18),(4,21),(4,24)$ respectively, subject to the single relation $e_{0}^{2}=d_{0} g$. That is the elements $e_{0}^{i} d_{0}^{j} g^{k}$ with $i=0,1, j \geqq 0, k \geqq 0$ are linearly independent.

The line of proof is as follows: Let $B$ be the exterior subalgebra of $A$ generated by $S q^{0,1}$ and $S q^{0,2}$. Then $H^{* *}(B)$ is a polynomial algebra on two generators, namely $x=\left\{\left[\xi_{2}\right]\right\}$ and $y=\left\{\left[\xi_{2}^{2}\right]\right\}$ in Milnor's [18] notation. $B$ has a basis consisting of the elements $S q^{0, j}$, where $0 \leqq j \leqq 3$. Hence $B^{*}$ has a basis consisting of the elements $\xi_{2}^{j}$ with $0 \leqq j \leqq 3$. The inclusion map $i: B \rightarrow A$ induces a map $i^{* *}: H^{* *}(A) \rightarrow H^{* *}(B)$. The proof depends on showing that $i^{* *} d_{0}=x^{2} y^{2}, i^{* *} e_{0}=x y^{3}, i^{* *} g=y^{4}$. This will show that the elements $e_{0}^{i} d_{0}^{j} g^{k}$ with $i=0,1, j \geqq 0, k \geqq 0$ are linearly independent. To obtain the relation $e_{0}^{2}=d_{0} g$ we observe that, by the above argument $e_{0}^{2}$ and $d_{0} g$ are both nonzero elements of $H^{8,42}(A) \cong Z_{2}$ (see May [12], Appendix $A$ ). Thus $e_{0}^{2}=d_{0} g$. This proves the theorem. It remains only to sketch how to compute the effect of $i^{* *}$ on $d_{0}, e, g$. The inclusion $i: B \rightarrow A$ induces a known map $i^{*}: A^{*} \rightarrow B^{*}$ of the dual algebras and a map $F\left(i^{*}\right): F\left(A^{*}\right) \rightarrow F\left(B^{*}\right)$ of the cobar construction. Now $F\left(i^{*}\right)$ maps the basis elements of $B^{*}$ to themselves and every other element to zero. From the explicit construction of cocycles $\tilde{d}_{0}, \tilde{e}_{0}, \tilde{g}$ re- 
presenting $d_{0}, e_{0}, g$ respectively it will follow that: $\left\{F\left(i^{*}\right) \tilde{d}_{0}\right\}=x^{2} y^{2}$, $\left\{F\left(i^{*}\right) \tilde{e}_{0}\right\}=x y^{3}$ and $\left\{F\left(i^{*}\right) \tilde{g}\right\}=y^{4}$, which completes the proof of the theorem, having in mind that $H^{4,18}(A)=H^{4,21}(A)=H^{4,24}(A) \cong Z_{2}$. Actually we use the following Massey products:

$$
\begin{aligned}
& d_{0}=\left\langle h_{2}^{2}, h_{0}, h_{2}^{2}, h_{0}>\right. \\
& e_{0}=<h_{3}^{2}, h_{0}^{2}, h_{1}, h_{0}> \\
& g=<h_{3}^{2} h_{0}, h_{0}, h_{1}, h_{2}>.
\end{aligned}
$$

\section{Cup-i-products}

Let $A$ be a connected cocommutative Hopf algebra (over $Z_{2}$, for simplicity) and $H(A)$ its cohomology; for instance-the $\bmod 2$ Steenrod algebra is such a Hopf algebra (see Milnor [18]). Let $F\left(A^{*}\right)$ be the Adams cobar construction. It follows from Adams work (see e.g. [2]) that there exist maps

$$
F\left(A^{*}\right) \otimes F\left(A^{*}\right) \stackrel{\cup_{i}}{\longrightarrow} F\left(A^{*}\right), \quad i=0,1,2 \ldots
$$

which have most of the properties of the Steenrod cup-i-products (see Steenrod [20]). The above cup-i-products induce Steenrod squares

$$
S q^{i}: H^{s, t}(A) \longrightarrow H^{s+i, 2 t}(A),
$$

which enjoy most of the properties of their topological analogues. (Cartan formula, Adem relation etc.) These operations and their applications have been studied by many authors. (See: Adams [2], Ivanovskii [6], Liulevicius [7], May [14], Milgram [15, 16, 17], Novikov [19]). The present writer has obtained explicit formulae for these cup-i-products in 1965, which appeared in his M.Sc. thesis [23], written under the supervision of Professor Adams. The detailed contents of [23] will appear elsewhere. Here we quote from $[23,25]$ the explicit formulae that we will need. More precisely: Let $x=\left[\alpha_{1}|\cdots| \alpha_{p}\right], y=\left[\beta_{1}|\cdots| \beta_{q}\right]$ be two cochains in $F\left(A^{*}\right)$. Then $x \cup_{i} y$ is described in terms of appropriate iterated diagonals of $A^{*}$, as follows:

Ist case: $i=2 t$ (even). The general summand of $x \cup_{i} y$ is: 


$$
\begin{aligned}
& {\left[\alpha_{1}|\cdots| \alpha_{r_{0}}\left|\alpha_{r_{0}+1}^{(1)} \beta_{1}\right| \cdots\left|\alpha_{r_{0}+1}^{\left(s_{1}-s_{0}-1\right)} \beta_{s_{1}-1}\right| \alpha_{r_{0}+2} \beta_{s_{1}}^{(1)}|\cdots| \alpha_{r_{1}} \beta_{s_{1}}^{\left(r_{1}-r_{0}-1\right)} \mid\right.} \\
& \cdots\left|\alpha_{r_{t-1}+1}^{(1)} \beta_{s_{t-1}+1}\right| \cdots\left|\alpha_{r_{t-1}+1}^{\left(s_{t}-s_{t-1}-1\right)} \beta_{s_{t}-1}\right| \alpha_{r_{t-1}+2} \beta_{s_{t}}^{(1)}|\cdots| \alpha_{r_{t}} \beta_{s_{t}}^{\left(r_{t}-r_{t-1}-1\right)} \\
& \left.\left|\beta_{s_{t}+1}\right| \cdots \mid \beta_{q}\right] .
\end{aligned}
$$

Actually $x \cup_{i} y$ is a sum of terms of this form depending on indices $j_{0}$, $j_{1}, \ldots, j_{i}$ subject to the relations:

$$
0 \leqq j_{0}<j_{1}<\cdots<j_{i} \leqq p+q-i ; t+\sum_{0 \leqq k \leqq i}(-1)^{k} j_{k}=p .
$$

The indices $r_{0}, r_{1}, \ldots, r_{t}$ and $s_{0}, s_{1}, \ldots, s_{t}$ are given by the equations:

$$
\begin{aligned}
& r_{m}=m+\sum_{0 \leqq k \leqq 2 m}(-1)^{k} j_{k}, s_{m}=m+\sum_{0 \leqq k \leqq 2 m-1}(-1)^{k+1} j_{k} \\
& \text { for } m=0,1, \ldots, t .
\end{aligned}
$$

Also: $s_{0}=0, r_{0}=j_{0}$ and $r_{t}=p$.

2nd case: $i=2 t-1$ (odd). The general summand of $x \cup_{i} y$ is:

$$
\begin{aligned}
& {\left[\alpha_{1}|\cdots| \alpha_{r_{0}}\left|\alpha_{r_{0}+1}^{(1)} \beta_{1}\right| \cdots\left|\alpha_{r_{0}+1}^{\left(s_{1}-s_{0}-1\right)} \beta_{s_{1}-1}\right| \alpha_{r_{0}+2} \beta_{s_{1}}^{(1)}|\cdots| \alpha_{r_{1}} \beta_{s_{1}}^{\left(r_{1}-r_{0}-1\right)} \mid\right.} \\
& \cdots\left|\alpha_{r_{t-2}+2} \beta_{s_{t-1}}^{(1)}\right| \cdots\left|\alpha_{r_{t-1}} \beta_{s_{t-1}}^{\left(r_{t}-r_{t-1}-1\right)}\right| \alpha_{r_{t-1}+1}^{(1)} \beta_{s_{t-1}+1}|\cdots| \alpha_{r_{t-1}+1}^{\left(s_{t}-s t-1\right)} \beta_{s_{t}-1} \mid \\
& \left.\alpha_{r_{t-1}+2}|\cdots| \alpha_{p}\right] .
\end{aligned}
$$

In this case $x \cup_{i} y$ is a sum of terms of this form depending on indices $j_{0}, j_{1}, \ldots, j_{i}$ subject to the relations:

$$
0 \leqq j_{0}<j_{1}<\cdots<j_{i} \leqq p+q-i ; t+\sum_{0 \leqq k \leqq i}(-1)^{k+1} j_{k}=q+1 .
$$

the indices $r_{0}, r_{1}, \ldots, r_{t}$ and $s_{0}, s_{1}, \ldots, s_{t}$ are given by the equations:

$$
\begin{aligned}
& r_{m}=m+\sum_{0 \leqq k \leqq 2 m}(-1)^{k} j_{k} ; s_{m}=\sum_{0 \leqq k \leqq 2 m-1}(-1)^{k+1} j_{k} \\
& \text { for } m=0,1, \ldots, t-1 .
\end{aligned}
$$

Also: $s_{0}=0, r_{0}=j_{0}$ and $s_{t}=q+1$.

Examples: For $x=\left[\alpha_{1}|\cdots| \alpha_{p}\right], y=\left[\beta_{1}|\cdots| \beta_{q}\right]$ we have: $x \cup y=\left[\alpha_{1}|\cdots| \alpha_{p}\left|\beta_{1}\right| \cdots \mid \beta_{q}\right]$. We write $x y$ for $x \cup y$. 


$$
\begin{aligned}
& \left.x \cup_{1} y=\sum_{0 \leqq j \leqq p-1}\left|\alpha_{1}\right| \cdots\left|\alpha_{j}\right| \alpha_{j+1}^{(1)} \beta_{1}|\cdots| \alpha_{j+1}^{(q)} \beta_{q}\left|\alpha_{j+2}\right| \cdots \mid \alpha_{p}\right] . \\
& x \cup_{2} y=\sum\left[\alpha_{1}|\cdots| \alpha_{j}\left|\alpha_{j+1}^{(1)} \beta_{1}\right| \cdots\left|\alpha_{j+1}^{(k-j)} \beta_{k-j}\right| \alpha_{j+2} \beta_{k-j+1}^{(1)}|\cdots| \alpha_{p} \beta_{k-j+1}^{(l-k)} \mid\right. \\
& \left.\beta_{k-j+2}|\cdots| \beta_{q}\right] .
\end{aligned}
$$

This summation is taken over all indices $j, k, l$ such that: $0 \leqq j<k<l \leqq$ $p+q-2 ; j-k+l=p-1$.

If $\delta$ is the coboundary of $F\left(A^{*}\right)$ then we have the usual coboundary formulae for cochains:

$$
\begin{aligned}
& \delta(x \cup y)=\delta x \cup y+x \cup \delta y \quad \text { and } \\
& \delta\left(x \cup_{i} y\right)=\delta x \cup_{i} y+x \cup_{i} \delta y+x \cup_{i-1} y+y \cup_{i-1} x \quad \text { for } i>0 .
\end{aligned}
$$

We also have Hirsch formulae:

$$
\begin{gathered}
\left(x_{1} \ldots x_{n}\right) \cup{ }_{1} z=\sum_{1 \leqq k \leqq n-1} x_{1} \ldots x_{k-1}\left(x_{k} \cup_{i} z\right) x_{k+1} \ldots x_{n} \quad \text { in } F\left(A^{*}\right) . \\
\text { e.g. } \quad(x y) \cup_{1} z=\left(x \cup_{1} z\right) y+x\left(y \cup_{1} z\right) . \\
<x, y, x>=\left(x \cup_{1} x\right) y \quad \text { in } H^{* *}(A)
\end{gathered}
$$

(modulo appropriate indeterminacies).

For the topological analogues of the last two equations see Hirsch [5].

\section{The Generators $d_{0}, e_{0}, g$.}

We will construct cocycles $\tilde{d}_{0}, \tilde{e}_{0}, \tilde{g}$ representing $d_{0}=<h_{2}^{2}, h_{0}, h_{2}^{2}$, $h_{0}>, e_{0}=<h_{3}^{2}, h_{0}^{2}, h_{1}, h_{0}>, g=<h_{3}^{2} h_{0}, h_{0}, h_{1}, h_{2}>$ respectively.

It is not difficult to see that these quadruple Massey products are defined.

\section{The cocycle $\tilde{\boldsymbol{d}}_{0}$.}

We may take:

$$
\tilde{d}_{0}=R \cup\left[\xi_{1}\right]+\left[\xi_{1}^{4} \mid \xi_{1}^{4}\right] \cup S+X X,
$$

where: 


$$
\begin{aligned}
& R=\left[\xi_{2}^{4}\left|\xi_{2}^{4}\right| \xi_{1}\right]+\left[\xi_{1}^{4}\left|\xi_{2}^{4}+\xi_{1}^{12}\right| \xi_{1}\right] ; S=\left[\xi_{2}^{2}+\xi_{1}^{6} \mid \xi_{1}^{4}\right] ; \\
& X=\left[\xi_{2}^{2} \mid \xi_{2}+\xi_{1}^{3}\right]+\left[\xi_{1}^{4} \mid \xi_{1}^{2} \xi_{2}\right]+\left[\xi_{3} \mid \xi_{1}^{2}\right] .
\end{aligned}
$$

The cocycle $\tilde{e}_{0}$.

We may take:

$$
\tilde{e}_{0}=R \cup\left[\xi_{1}\right]+\left[\xi_{1}^{8} \mid \xi_{1}^{8}\right] \cup S+X Z,
$$

where now $R, S$ are such that $\delta R, \delta S$ give representative cocycles for $\left.<h_{3}^{2}, h_{0}^{2}, h_{1}\right\rangle,\left\langle h_{0}^{2}, h_{1}, h_{0}>\right.$ respectively. Also $X, Z$ are such that $\delta X$, $\delta Z$ give representative cocycles for $h_{3}^{2} h_{0}^{2}, h_{1} h_{0}$ respectively.

\section{The cocycle $\tilde{\mathbf{g}}$.}

We may take:

$$
\tilde{g}=\left[\xi_{1}^{8}\left|\xi_{1}^{8}\right| \xi_{1}\right] \cup R+S \cup\left[\xi_{1}^{4}\right]+X Z \text {. }
$$

Here $R, S$ are such that $\delta R, \delta S$ give representative cocycles for $<h_{0}, h_{1}$, $h_{2}>,\left\langle h_{3}^{2} h_{0}, h_{0}, h_{1}>\right.$ respectively and $X, Z$ are such that $\delta X, \delta Z$ give representative cocycles for $h_{3}^{2} h_{0}^{2}, h_{1} h_{2}$ respectively. By using the explicit formulae for the cup-i-products involved in these constructions it can be shown that:

$$
\begin{aligned}
& F\left(i^{*}\right) \tilde{d}_{0}=\left[\xi_{2}\left|\xi_{2}\right| \xi_{2}^{2} \mid \xi_{2}^{2}\right], \\
& F\left(i^{*}\right) \tilde{e}_{0}=\left[\xi_{2}\left|\xi_{2}^{2}\right| \xi_{2}^{2} \mid \xi_{2}^{2}\right], \\
& F\left(i^{*}\right) \tilde{g}=\left[\xi_{2}^{2}\left|\xi_{2}^{2}\right| \xi_{2}^{2} \mid \xi_{2}^{2}\right],
\end{aligned}
$$

as required.

The actual computations are lengthy and they are omitted here. Details can be found in [25], where $g$ is described in another way, based on a proposition which is crucial for proving the Adams periodicity theorem $[3]$.

\section{References}

[1] Adams, J.F., On the structure and applications of the Steenrod algebra, Comm. Math. Helvetici 32 (1958), 180-214. 
[2] - On the non-existence of elements of Hopf invariant one, Ann. of Math. 72 (1960), 20-104.

[3] - A periodicity theorem in homological algebra, Proc. Camb. Phil. Soc. 62 (1966), 365-377.

[4] - Stable Homotopy Theory, 3rd ed., Lecture Notes in Math., no. 3, Springer-Verlag, 1969.

[5] Hirsch, G.H., Quelques propriétés des produits de Steenrod, C. R. Acad. Sci. (Paris) 241, pt. 2, (1955), 923-925.

[6] Ivanovski, L. N., Cohomology of the Steenrod algebra, Dokl. Akad. Nauk. SSSR 157 (1964), 1284-1287.

[7] Liulevicius, A.L., The factorisation of cyclic reduced powers by secondary cohomology operations, Mem. of Amer. Math. Soc. 42, 1962.

[8] - Coalgebras, resolutions and the computer, University of Chicago, 1964 (mimeographed).

[9] Mahowald, M., and M.C. Tangora, An infinite subalgebra of $\operatorname{Ext}_{A}^{* *}\left(Z_{2}, Z_{2}\right)$, Trans. Amer. Math. Soc. 132 (1968), 263-274.

[10] Margolis, H., S. Priddy and M. Tangora, Another Systematic phenomenon in the cohomology of the Steenrod algebra, Topology 10 (1971), 43-46.

[11] Margolis H.R., The cohomology of finite algebras (to appear).

[12] May, J.P., The cohomology of restricted Lie algebras, etc., Ph.D. Thesis, Princeton University, 1964.

[13] - The cohomology of the Steenrod algebra; stable homotopy groups of spheres, Bull. Amer. Math. Soc. 71 (1965), 377-380.

[14] — A general algebraic approach to Steenrod operations, The Steenrod algebra and its applications. (A conference to celebrate N.E. Steenrod's sixtieth birthday), Lecture Notes in Math. no. 168, Springer-Verlag, 1970. 153-231.

[15] Milgram, R.J., Steenrod squares and higher Massey products, Bol. de la Soc. Mat. Mexicana (1968), 32-57.

[16] - Symmetries and operations in Homotopy Theory, Amer. Math. Soc., Summer Institute on Algebraic Topology held at the University of Wisconsin, 1970.

[17] $\longrightarrow$, Group representations and the Adams spectral sequence (to appear).

[18] Milnor, J.W., The Steenrod algebra and its dual, Ann. of Math. 67 (1958), 150171.

[19] Novikov, S.P., On the cohomology of the Steenrod algebra, Dokl. Akad. Nauk SSSR 128 (1959), 893-895.

[20] Steenrod, N.E., Products of cocycles and extensions of mappings, Ann. of Math. 48 (1947), 290-320.

[21] Tangora, M.C., On the cohomology of the Steenrod algebra, Math. Zeitschrift 116 (1970), 18-64.

[22] Whitehead, G.W., Recent advances in homotopy theory, CBMS, no. 5, Amer. Math. Soc. 1970.

[23] Zachariou, A., On cup-i-products in the cobar construction $F\left(A^{*}\right)$, M. Sc. Thesis, University of Manchester, 1966.

[24] - A subalgebra of $\operatorname{Ext}_{A}^{* *}\left(Z_{2}, Z_{2}\right)$, Bull. Amer. Math. Soc. 73 (1967), 647648. 
[25] - Cohomology operations in the cobar construction-Applications, Ph.D. Thesis, University of Manchester, 1968.

Department of Mathematics

The Johns Hopkins University

Baltimore, Maryland 21218

U.S. A. 